Rev. Saude públ., S. Paulo

$11: 502-9,1977$.

\title{
MODELO MATEMÁTICO PARA ESTIMAR IMPACTO EPIDEMIOLÓGICO DA VACINAÇÃO BCG
}

Antonio Ruffino-Netto*

Gilberto Ribeiro Arantes**

RSPU-B/382

Ruffino-Netto, A. \& ARANTES, G. R. Modelo matemático para estimar impacto epidemiológico da vacinação BCG. Rev. Saúde públ., S. Paulo, 11: 502-9, 1977.

RESUMO: Foi apresentado modelo que permite estimar o impacto primário de um programa de vacinaf̧ão com BCG intradérmico, aplicado num determinado grupo etário, durante um certo periodo de tempo, que se segue à vacinação. Algurs exemplos de aplicação do mesmo foram mostrados, tendo sido assinalada a possibilidade de estimar antecipadamente a menor eficácia de vacina capaz de manter a relação custo-beneficio maior do que um.

Unitermos: Tuberculose, modelos matemáticos. Vacinação BCG. Custobeneficio, análise.

\section{I N T RODUCB}

Modelo tem sido definido "como representação simbólica da vida real, evidentemente simplificado para permitir um tratamento lógico ou matemático"10. Consequentemente, nenhum modelo é capaz de sintetizar toda uma realidade (biopsicossocial), por isso deve ser entendido sempre coms instrumento de trabalho aberto a reformulações e adequações, à medida que novos conhecimentos sobre o assunto sejam descobertos ou evidenciados pela sua aplicação prática.

Se por um lado, longe está a pretensão do domínio de toda a realidade apontada, por outro lado, esforços deverão ser feitos no sentido de quantificar relações entre alguns dos eventos que constituem parte ("parcela muito pequena") dessa realidade.
A formulação de modelos contribui para o aperfeiçoamento daqueles que militam em qualquer área de atividades, especialmente no campo da saúde, no sentido de substituir o hábito de decidir, baseados em meras suposições quando se trata da aplicação de recursos com o objetivo de beneficiar uma determinada coletividade.

Vários autores têm apresentado outros modelos, com definições mais ou menos superponíveis. Objetivos, méritos, etapas para sua construção, tipos e parâmetros envolvidos (demográficos, epidemiológicos, operacionais, técnicos, sociais e econômicos) na formulação dos modelos têm sido evidenciados e discutidos $3,5,7,8,9,10,11,12$.

Alguns têm distinguido entre modelos de simulação e modelos de decisão ${ }^{3}$. Eviden-

* Da Faculdade de Medicina de Ribeirão Preto da USP - Ribeirão Preto. S.P. - Brasil.

* Da Divisão de Estudos e Programas da Coordenadoria de Saúde da Comunidade da Secretaria da Saúde do Estado de São Paulo. Ar. São Luiz, 99 - São Paulo, SP Brasil. 
RUFFINO-NETTO, A. \& ARANTES, G. R. Modelo matemático para estimar impacto epidemiológico da vacinação BCG. Rev. Saúde públ., S. Paulo, 11:502-9, 1977.

temente, os modelos de simulação terão um poder preditivo tanto maior quanto maior for a sensibilidade e a especificidade dos fatores envolvidos na alimentação dos mesmos.

$\mathrm{Na}$ fundamentação dos programas de controle da tuberculose, tem-se sugerido normas técnicas e administrativas 2,6 . Conhecimentos recentes na área da matemática (como a programação linear por exemplo) têm sido utilizados em modelos de decisão no controle da tuberculose *.

No presente trabalho, temos por objetivo montar um modelo que permita prever o impacto de um programa de vacinação em certo grupo etário durante certo periodo, quando o programa for aplicado no grupo etário imediatamente mais jovem.

\section{MODELO}

Definindo-se :

Go = determinado grupo etário

Po $=$ população do grupo Go

$\mathrm{G}=$ outro grupo etário, mais velho e não superponível ao grupo Go

$\mathrm{h}=$ intervalo dos grupos Go-G

$P=$ população do grupo $G$

$\mathrm{k}=$ taxa constante de morbidade, suposta uniforme no grupo $G$

$\mathrm{p}$ = cobertura do programa na população a ser vacinada (sendo $0 \leqslant p \leqslant 1$ )

$\mathrm{E}=$ eficácia da vacina utilizada no ponto médio de $h$ (sendo $0 \leqslant \mathrm{E}$ $\leqslant 1)$

$$
\begin{aligned}
c_{1}= & \text { custo da vacinação por pessoa } \\
c_{.2}= & \text { custo do tratamento quimoterápico } \\
& \text { da tuberculose. }
\end{aligned}
$$

Problema: Qual seria a redução do problema da tuberculose no grupo G, nos próximos $n$ anos consecutivos a um suposto programa de vacinação aplicado atualmente no Grupo Go, com meta de cobertura $(\mathrm{p} \times 100) \%$, utilizando-se uma vacina com eficácia $(E x$ 100) $\%$.

\section{SOLUÇÃO DO MODELO}

A magnitude do problema no grupo $G$ será dada pela incidência esperada entre os vacinados que ano a ano passam para esse grupo etário, somada à incidência esperada entre os não vacinados que ainda não o deixaram.

Supondo-se que $\mathrm{n}$ seja igual a $\mathrm{h}$ e que a população $P$ seja uniformemente distribuida em $G$, o modelo será assim solucionado:

a) Total de casos esperados de tuberculose no grupo $G$, na ausência de vacinação no grupo Go:

$$
\mathrm{T}_{1}=\mathrm{P} \cdot \mathrm{k} \cdot \mathrm{n}
$$

b) Total de casos esperados de tuberculose no grupo G, com programa de vacinação com cobertura $(\mathrm{p} \times 100) \%$ do grupo Go, vacina com eficácia $(E \times 100) \%$ :

$$
\begin{gathered}
T_{2}=\frac{P}{n} k(1-E) p(1+2+\ldots+n)+\frac{P}{n} k(1-p)(1+2+\ldots+n)+ \\
\frac{P}{n} k[(n-1)+(n-2)+\ldots 2+1+0]
\end{gathered}
$$

ou seja:

$$
\begin{gathered}
\mathrm{T}_{:}=\frac{\mathrm{P}}{\mathrm{n}} \mathrm{k}(1+2+\ldots+\mathrm{n})[(1-\mathrm{E}) \mathrm{p}+(1-\mathrm{p})]+\frac{\mathrm{P}}{\mathrm{n}} \mathrm{k} \\
{[0+1+2+\ldots+(\mathrm{n}-1)]}
\end{gathered}
$$


RUFFINO-NETTO, A. \& ARANTES, G. R. Modelo matemático para estimar impacto epidemiológico da vacinação BCG. Rer. saúde pribl., S. Paulo, 11:502-9, 1977.

Observar que na expressão (3) aparecem que a soma de n termos na PA será dada 2 progressões aritméticas (PA), de razão por: $(\mathrm{r})=1$, sendo que uma apresenta $01^{\text {" }}$ termo $a_{1}=1$ e outra $a_{1}=0$ Lembrando

$$
\mathrm{S}_{\mathrm{n}}=\mathrm{na}_{1}+\frac{\mathrm{n}(\mathrm{n}-1) \mathrm{r}}{2}(4)
$$

yue substituida e simplificada na expressão (3), resulta:

$$
\begin{aligned}
& \begin{aligned}
T_{0}=\frac{P}{n} k[n & \left.\left.+\frac{n(n-1)}{2}\right] \mid(1-E) p+(1-p)\right]+ \\
+ & -\frac{P}{n}\left|\frac{n-1)}{2}\right|
\end{aligned} \\
& T_{.,}=P_{k}\left[1+\frac{(1-1)}{2}\right][(1-E) p+(1-p)]+P k\left[\frac{(n-1)}{2}\right] \\
& T_{n}=P k\left[\left(1+\frac{(n-1)}{2}\right)((1-E) p+(1-p))+\frac{(n-1)}{2}\right]
\end{aligned}
$$

A redução (R) (em numero absoluto) de casos da doença no grupo G, será dado por:

$$
\mathrm{R}=\mathrm{T}_{1}-\mathrm{T}_{2}
$$

(1) e (7) substituiclas em (8), resulta:

$$
\begin{array}{r}
R=P k n-P k\left[\left(1+\frac{(n-1)}{2}\right)((1-E) P+(1-p))+\frac{(n-1)}{2}\right] \\
R=P k \quad\left[n-\left(1+\frac{(n-1)}{2}\right)((1-E) P+(1-p))-\frac{(n-1)}{2}\right]
\end{array}
$$

oll

$$
R=P k\left[\left(\frac{n+1}{2}\right)-\left(1+\frac{(n-1)}{2}\right)((1-E) p+(1-n))\right]
$$

Esta expressão fornecerá, portanto, o valor da redução do problema (em nủmero absoluto) no grupo $G$ considerado. $O$ custo total do programa de vacinação (V) no grupo Go, será dado por:

$$
V=\text { Po.p.c. }
$$

Como houve uma redução dos casos de tuberculose no grupo G, certa quantidade de recursos deixou de ser utilizada, significando, portanto, dinheiro poupado; este dinheiro poupado, que chamaremos de $D$, será dado por:

$$
\mathrm{D}=\mathrm{R} \cdot \mathrm{C} .
$$

ou seja

$$
D=C_{2} P k\left[\left(\frac{n+1}{2}\right)-\left(1+\frac{(n-1)}{2}\right)((1-E) p+(1-p))\right]
$$

A comparação das expressões (12) e (14), ou seja, entre V e D evidenciará os beneficios decorrentes. Assim, se D é maior que $V$, isto significa que apenas com o beneficio primário da vacinação já compensa o gasto com o programa efetuado, 
RUFFINO-NETTO, A. \& ARANTES, G. R. Modelo matemático para estimar impacto epidemiológico da vacinação BCG. Rev. Saúde públ., S. Paulo, 11:502-9, 1977.

sendo que $(\mathrm{D}-\mathrm{V})$ é igual à economia; por outro lado $\mathrm{D} / \mathrm{V}$ fornecerá a relação da quantidade de dinheiro (cruzeiros) economizado por dinheiro gasto.

\section{EXEMPLOS}

Usando dados aproximados para o Estado de São Paulo, onde temos:

$$
\begin{aligned}
& \text { Go = grupo etário } 0-14 \text { anos } \\
& P_{0}=8.000 .000 \quad \text { (populaçãu } \quad 0 \quad \ldots \quad 14 \\
& \text { anos) } \\
& \mathrm{G}=\text { grupo etário } 15-29 \text { anos } \\
& \mathrm{P}=6.000 .000 \text { (população } 15-29 \\
& \text { anos) } \\
& \mathrm{h}=15 \text { (intervalu } 0-14 \text { e } 15-29 \\
& \text { anos) } \\
& k=0,0003 \text { oll seja } 0,3 \% \text { * * }
\end{aligned}
$$

e supondo os seguintes custos (a título de exemplo) :

$$
\mathrm{C}_{1}=\underset{\text { na aplicada) }}{1,00 \text { (custo em cruzeiros da vaci- }}
$$

$\mathrm{C}_{2}=1.000,00$ (custo en cruzeiros do tratamento), apresentamos alguns exemplos:

4.1. Suponhamos que um programa de vacinação (vacina de eficácia $80 \%$, isto é, $E=0,8$ ) seja aplicado no atual grupo de $0-14$ anos, com meta de cobertura igual a $80 \%$ (ou seja $\mathrm{p}=0,8$ ). Estudar redução do problema da tuberculose nos próximos 15 anos $(n=15)$ no grupo etário $15-29$ anos.

a) Total de casos esperados na ausência de vacinação:

(1) $T_{1}=$ P.k.n

$\mathrm{T}_{1}=0.000 .000 \times 0,0003 \times 15=27.000$

b) Total de casos esperados sob ação do programa:

$$
\begin{aligned}
& \text { (7) } \mathrm{T}_{2}=\mathrm{Pk}\left[\left(1+\frac{(\mathrm{n}-1)}{2}\right)((1-\mathrm{E}) \mathrm{p}+(1-\mathrm{p}))+\frac{(\mathrm{n}-1)}{2}\right] \\
& \mathrm{T}_{2}=6.000 .000 \times 0,0003\left[\left(1+\frac{(15-1)}{2}\right)((1-0,8) 0,8+(1-0,8))+\frac{15-1}{2}\right] \\
& \mathrm{T}_{.,}=1800[8(0,16+0,2)+7] \\
& \mathrm{T}=1800 \times 9,88=17.784 \\
& R=T_{1}-T_{2}=27.000-17.784=9.216 \\
& \mathrm{R}=\operatorname{Pk}\left[\frac{\mathrm{n}+1}{2}-\left(1+\frac{(\mathrm{n}-1)}{2}\right)((1-\mathrm{E}) \mathrm{p}+(1-\mathrm{p}))\right] \\
& R=6.000 .000 \times 0,0003\left[\frac{15+1}{2}-\left(1+\frac{15-1}{2}\right)((1-0,8) 0,8+(1-0,8))\right] \\
& R=1800[8-8(0,16+0,2)]=9216 \\
& \text { O custo de vacinação será } \\
& \mathrm{V}=\text { Po.p. } \mathrm{C}_{1}=8.000 \cdot 0 \mathrm{C} 0 \times 0,8 \times 1=\mathrm{Cr} \$ 6.400 \cdot 000,00 \\
& O \text { dinheiro poupado será } \\
& D-\text { R.C }_{2}=9.215 \times 1000=\operatorname{Cr} \$ 9.216 .000,00
\end{aligned}
$$

* Estimativa feita no Centro de Saúle de Ribeirão Preto. 
RUFFINO-NETTO, A. \& ARANTES, G. R. Modelo matemático para estimar impacto epidemiológico da vacinação BCG. Rev. Saúde publ., S. Paulo, 11:502-9, 1977.

Observamos que a economia (D) é maior que o gasto (V); assim, a quantidade de dinheiro (cruzeiros) economizado por cruzeiro gasto será $\frac{\mathrm{D}}{\mathrm{V}}=\frac{9.216 .000}{6.400 .000}=\operatorname{Cr} \$ 1,44$

4.2. Con os mesmos dados do Estado de São Paulo e os mesmos custos supostos, simulamos diferentes combinações de programas de vacinação (com vacinas de eficácias diferentes e/ou coberturas diferentes), e obtivemos os resultados apresentados na Tabela. Observamos que a diferença (D-V) aumenta proporcionalmente em função de $p$ para as vacinas com eficácia $E=0,6$ e $E=0,8$, o mesno não ocorrendo com a vacina de eficácia $E=0,4$ (Figura).

Por outro lado, observamos ainda nessa Tabela que a relação $\mathrm{D} / \mathrm{V}$ (unidade de dinheiro economizado por uniclade de dinheiro gasto), independe de $p$, sendo função apenas da eficácia da vacina $E$.

Frente a esta situação perguntamos qual seria a menor eficácia de vacina que seria capaz de manter a relação

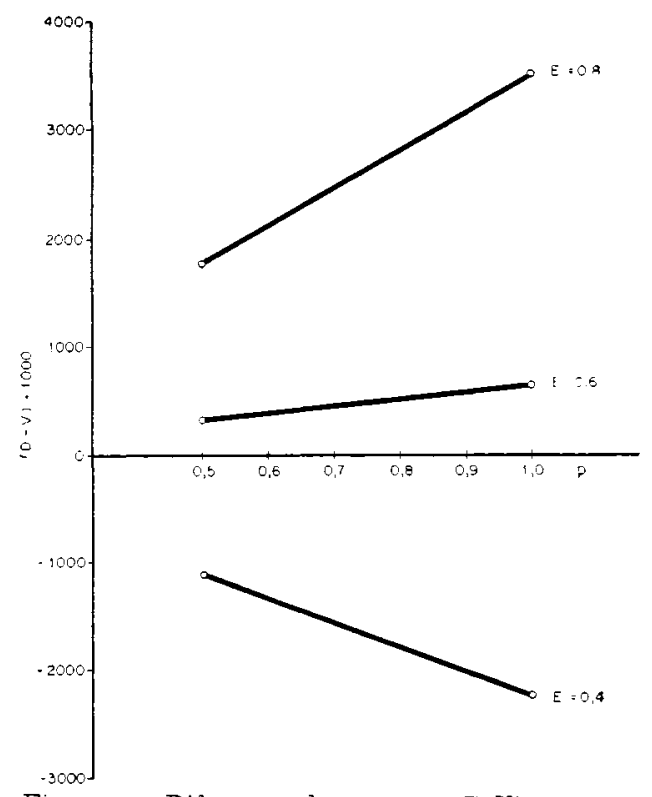

Figura - Diferença dos custos (D-V) segundo diferentes coberturas (p) de programa de racinação $B C G$.

$D-V \geqslant 0$, ol o que seria equivalente, $\frac{\mathrm{D}}{\mathrm{V}} \geqslant 1$

A solução será dada por:

$\frac{D}{V}=\frac{C{ }_{2} P k\left[\frac{(n+1)}{2}-\left(1+\frac{n-1}{2}\right)((1-E) p+(1-p))\right]}{P_{0} \cdot C_{1}} \geqslant 1$

ou

$\mathrm{C}_{2} \mathrm{Pk}\left\lceil\left(\frac{\mathrm{n}+1}{2}\right)-\left(1+\frac{\mathrm{n}-1}{2}\right)((1-\mathrm{E}) \mathrm{p}+(1-\mathrm{p}))\right] \geqslant$ Po.p.C

Com os dados estimados para o Estado de São Paulo, encontramos que a menor eficácia de vacina capaz de satisfazer essa condição seria $\mathrm{E}=0,555$.

Isto significa que, num programa de vacinação (qualquer que seja a meta de cobertura), o dinheiro poupado (D) será sempre maior que o custo de vacinação $(V)$, se a eficácia da vacina for no mínimo de $55,5 \%$.

\section{COMENTARIOS}

$O$ fato de se supor uniforme a morbidade no grupo seguinte, não invalida a sua aplicação porque o objetivo é prever o impacto nesse grupo como um todo, isto é, o número total de casos evitados como efeito primário da vacinação; aliás é preciso esclarecer também que neste modelo sim. 


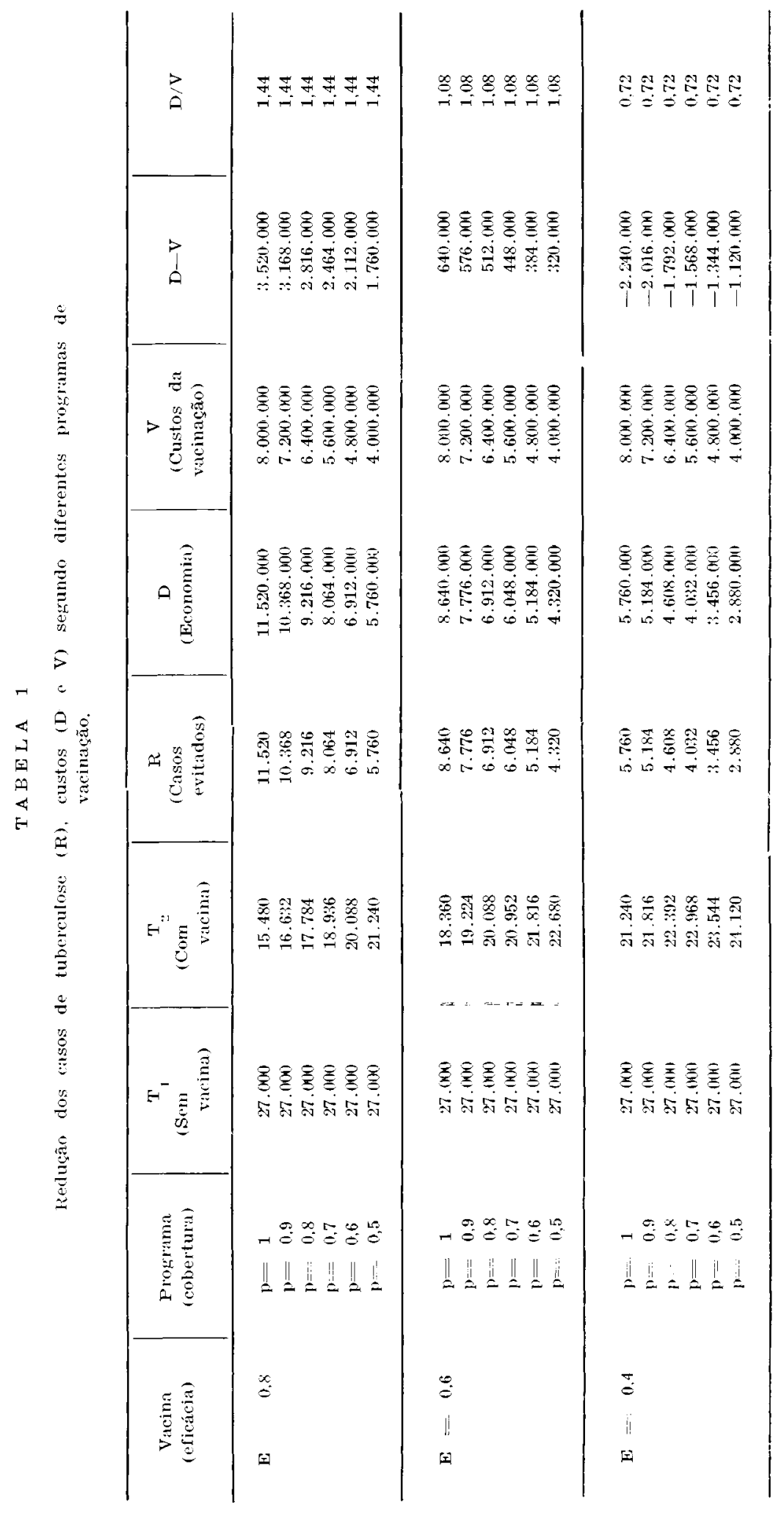


RUFFINO-NETTO, A. \& ARANTES, G. R. Modelo matemático para estimar impacto epidemiológico da vacinação BCG. Rev. Saúde públ., S. Paulo, 11:502-9, 1977.

ples a previsão não considera o impacto secundário, ou seja, aquele decorrente de outras infecções e casos que deixarão de ocorrer em conseguiència da não ocorrência dos primeiros.

Obviamente os resultados obtidos nos exemplos apresentados não têm a pretensão de oferecer elementos para decisão quanto a área em tela uma vez que os dados usados, principalmente as estimativas de custos, devem ser diferentes dos reais. Assim, se u custo "per capita" da vacinação fosse o dobro ou o triplo do valor usado, D $\mathrm{V}$ talvez fosse negativo e nesse caso o quociente $\mathrm{D} / \mathrm{V}$ não seria compensador em termos puramente econômicos. É conveniente ressaltar também que, aplicado as grupo etário 0 a 14 anus para previsão de impacto primário no grupo 15 a 29 anos, é válido afirmar que a relação $D / V$ não sofrerá influências com a variação das metas de cobertura. Possivelmente, tratando-se de outros grupos etários, ou no caso da população considerada como um todo, conforme a expectativa de incidência e a propurção de vacináveis (não infectados), variações na cobertura poderão influir na relação custo-benefício ${ }^{1}$.

\section{CONCLUSOES}

1. A partir de um modelo relativamente simples é possivel estimar o impacto primário esperado e a razão custo-benefício de programas de vacinação $B C(j$.

2. Esse modelo alimentado com dados reais poderá fornecer informações úteis para a tomada de decisões referentes à execução de programa de vacinação $B C G$, em uma determinada área.

RufFino-NETTO, A. \& ARANTES, G. R. [A mathematical model for estimating the effectiveness of $B C G$-vacicination] Rev. Saúde públ., $S$. Paulo, 11: $502-9,1977$.

ABSTRACT: A model was divised that estimates the primary impact of an immunization programme with intradermal $B C G$, applied to an age-group, during a certain period of time following vaccination. Some examples of its application show the possibility of estimating previously the least effectiveness of the vaccine which is able to maintain the relationship cost-benefit greater than 1.

UNITERMS: Tuberculosis, epidemiometric models. BCG vaccination. Costbenefit analysis.

\section{REFERENCIAS BIBLIOGRAFICAS}

1. ARANTES, G. R. Planejamento de atividades anti-tuberculose pelo método CENDES/OPS. Rev. Saude públ., S. Paulo, 10:17-29, 1976.

2. PIO, A. Normas tecnicas y administrativas para elaborar e implementar programas de tuberculosis. Ginebra, Organizacion Mundial de la Salud, 1975. (WHO $-\mathrm{CD} / \mathrm{TB} / 5)$.
3. PIOT, M. A. A simulation model of case finding and treatment in tuberculosis control programmes. Geneva, World Health Organization, 1967. (WHO/TB/ Technical Information/67.53).

4. PIOT, M. \& SUNDARESAN, T. K. A linear programme decision model for tuberculosis control. Geneva, World Health Organization, 1967 (WHO/TB/Technical Information/67.65). 
RUFFINO-NETTO, A. \& ARANTES, G. R. Mndelo matemático para estimar impacto epidemiológico da vacinação BCG. Rev. Saúde públ., S. Paulo, 11:502-9, 1977.

5. RUFFINO-NETTO, A. Epidemiologia da tuberculose: estudo de alguns aspectos ligados a modelos de prevenção, diagnóstico e modelos epidemiométricos. Ribeirão Preto, SP, 1975. [Tese de livre docência - Faculdade de Medicina de Ribeirão Preto da USP].

6. STYBLO, K. \& SUTHERLAND, I. Epidemiological indices for planning, surveillance and evaluation of tuberculosis programmes. Bull. int. Un. Tuberc., $49: 66-73,1974$.

7. WAALER, H. T. Cost-benefit analysis of $B C G$ : vaccination under various epidemiological situations. In: INTERNATIONAL TUBERCULOSIS CONFERENCE, 19th, Amsterdam, 1967. Proceeaings. Amsterdam, Excerpta Medica Foundation, 1967. p. 42-52. (International Congress Series, 164).

8. WAALER, H. T. A dynamic model for the epidemiology of tuberculosis. Amer. Rev. resp. Dis., 98:591-600, 1968.
9. WAALER, H. T. Model simulation and decision - making in tuberculosis programmes. Bull. int. Un. Tuberc., 43:337-44, 1970 .

10. WAALER, H. T. \& PIOT, M. A. The use of an epidemiological model for estimating the effectiveness of tuberculosis control measures: sensitivity of the effectiveness of tuberculosis control measures to the Coverage of the population. Bull. Wld Hlth Org., 41:75-93, 1969 .

11. WAALER, H. T. et al. The use of mathematical models in the study of the epidemiology of tuberculosis. Amer. J. publ. Hlth, 52:1002-13, 1962.

12. WORLD HEALTH ORGANIZATION, Expert Committee on Tuberculosis, $A$ decision model for tuberculosis control. Geneva, 1973. (WHO/TB/WP 73.3).

Recebido para publicaşāo em 02/03/197\% Aprovado para publicaçāo em 28/03/197\% 